\title{
Hierarchy of Determinants Underlying Death among HIV-Infected Patients in French Guiana
}

\author{
Matthieu Hanf ${ }^{1,2 *}$, Antoine Adenis ${ }^{1}$, Bernard Carme ${ }^{1,2}$, Pierre Couppié ${ }^{2,3}$, and Mathieu Nacher ${ }^{1,2}$ \\ ${ }^{1}$ Centre d'Investigation Clinique Epidémiologie Clinique Antilles Guyane CIC-EC INSERM CIE 802, Cayenne General Hospital, Cayenne, French Guiana, France \\ ${ }^{2}$ Department of Parasitology and Mycology-Team EA 3593, Université des Antilles et de la Guyane, Cayenne, French Guiana, France
} ${ }^{3}$ Department of Dermatology, Cayenne General Hospital, Cayenne, French Guiana, France

\begin{abstract}
Objective: In order to determine how risk factors of death among HIV-infected patients in French Guiana interact with one another and to define high risk population segments, an alternative statistical method to Cox proportional hazards models was used.

Patients and methods: Data from HIV+ patients followed in the three main hospitals of French Guiana were used. Data were extracted from the prospective French Hospital Database for HIV (FHDH). To examine the nature of the relationships between time of death since the first consultation and a set of predictor variables (age at enrolment, gender, nationality, CD4 count at enrolment, presence or absence of HAART at enrolment, a declared addiction, and mode of acquisition of the virus) a survival tree analysis was used. Survival rates at 5 years and 10 years and their $95 \%$ confidence interval were also calculated.
\end{abstract}

Results: This analysis permitted to reveal a new specific subgroup of HIV+ patients particularly at risk in French Guiana not already mentioned in the literature: the HIV+ female patients with a declared addiction.

Conclusions: Prevention and care in this vulnerable segment of the HIV+ population identified by this methodology should be reinforced.

Keywords: HIV/AIDS; Mortality; Female; Addiction; Epidemiology; Risk factor; French Guiana; Tree based method

\section{Introduction}

French Guiana is the French overseas territory where the HIV epidemic is most prevalent. HIV prevalence among pregnant women is $1.5 \%$. AIDS incidence is 10 times greater than in metropolitan France [1]. Transmission occurs through heterosexual sex in over $90 \%$ of cases. The standards of healthcare are close to those of mainland France. Approximately three-quarters of HIV-infected patients are foreign citizens [1].

In French Guiana, a previous study using a single failure Cox proportional hazards model had identified the main risk factors for death among a cohort of HIV+ patients since the introduction of HAART (Highly Active Antiretroviral Therapy) [2]. In this study, having declared an addiction, age $>60$, male gender and CD4 count $<50$ cells/ $\mu \mathrm{L}$ were independently associated with death. However, it is still difficult to disentangle how these risk factors interact with one another and to define high risk HIV+ population segments which could be specifically targeted by public health interventions.

Tree-structured models for survival data is a nonparametric methodology that has the ability to efficiently segment populations into meaningful subgroups and thus could be a useful tool for this task. In this perspective, this study, by using tree-structured models for survival data, aimed to find which factors, and which combinations of factors, could be particularly predictive of death in view to determine specific HIV+ patient sub-groups the most at risk of early death in French Guiana.

\section{Patients and Methods}

HIV+ patients followed at the Cayenne, Saint Laurent du Maroni and Kourou General Hospitals between 1 January 1996 and 31 December 2007 were obtained from the French Hospital Database for HIV (FHDH). The FHDH is an ongoing prospective observational nationwide, hospital based cohort to which patients have been continuously recruited in 62 hospitals since 1992. The only FHDH inclusion criteria are HIV type 1 or 2 infection and written informed consent. Data are collected prospectively by trained research assistants using standardized forms. Demographic data are recorded at inclusion. A follow-up form is completed at least every 6 months or at each visit or hospital admission during which a new illness is diagnosed, a new treatment is prescribed, or a noteworthy change in biological markers is noted. Patient identity is encrypted before the data are sent to the Ministry of Health and the Institute National de la Recherche Médicale, which centralizes data from COREVIHs (Regional Coordination of the fight against HIV) throughout France. This data collection is approved by the Commission Nationale Informatique et Libertés.

The outcome of interest was time elapsed between the first consultation and death. The same explanatory variables as previous study were used. These were age at enrolment, gender, nationality (French citizens or non-French citizens), CD4 count at enrolment, presence or absence of HAART at enrolment, a declared addiction and mode of acquisition of the virus. The studied failure event was death. Right censoring occurred at the last visit.

*Corresponding author: Matthieu HANF, Centre d'Investigation Clinique Epidémiologie Clinique Antilles Guyane CIC-EC INSERM CIE 802, Cayenne General Hospital, EHPAD, Avenue des Flamboyants, BP 600697300 Cayenne, French Guiana, France, E-mail: matthieu@hanf.fr

Received August 28, 2012; Accepted September 24, 2012; Published Septembe 26, 2012

Citation: Hanf M, Adenis A, Carme B, Couppié P, Nacher M (2012) Hierarchy of Determinants Underlying Death among HIV-Infected Patients in French Guiana. AIDS Clinic Res 3:172. doi:10.4172/2155-6113.1000172

Copyright: (C) 2012 Hanf M, et al. This is an open-access article distributed unde the terms of the Creative Commons Attribution License, which permits unrestricted use, distribution, and reproduction in any medium, provided the original author and source are credited. 
Citation: Hanf M, Adenis A, Carme B, Couppié P, Nacher M (2012) Hierarchy of Determinants Underlying Death among HIV-Infected Patients in French Guiana. J AIDS Clinic Res 3:172. doi:10.4172/2155-6113.1000172

Page 2 of 3

\begin{tabular}{|l|c|c|}
\hline Variable & N & $\%$ \\
\hline Gender & & 52 \\
\hline Female & 891 & 48 \\
\hline Male & & \\
\hline Mode of acquisition of the virus & 1557 & 84 \\
\hline Heterosexual relation & 47 & 3 \\
\hline Homosexual/bisexual relation & 254 & 14 \\
\hline Others/unknown & & \\
\hline Nationality & 313 & 17 \\
\hline French citizens & 1110 & 60 \\
\hline Non-French citizens & 435 & 23 \\
\hline Unknown & 1723 & 93 \\
\hline having a declared addiction & 135 & 7 \\
\hline No & 75 & 56 \\
\hline Yes & 17 & 13 \\
\hline With at least alcohol & 67 & 50 \\
\hline With at least marijuana & & \\
\hline With at least crack cocaine & 1538 & 83 \\
\hline Having a HAART at enrolment & 320 & 17 \\
\hline No & & \\
\hline Yes & 1662 & 11 \\
\hline Death recorded in the database & 196 & 271 \\
\hline No & Mean (min - max) & Standard dev. \\
\hline Yes & $42(0-88)$ & \\
\hline Variables & $(1-1655)$ & \\
\hline Age at enrolment & & \\
\hline CD4 count at enrolment & & \\
\hline & & \\
\hline
\end{tabular}

To examine the nature of the relationships between time elapsed between the first consultation and death and its predictor variables, a survival tree analysis (STA) called unbiased recursive partitioning was used [3]. STA are ideally suited for the analysis of epidemiological data with hierarchical and complex relationships among the predictor variables [3]. It requires a statistically significant difference $(p<0.05)$, adjusted with Bonferroni correction, and a node size superior to 200 in order to partition the data, which minimizes bias and prevents over-fitting and the need for pruning. The party package within the $\mathrm{R}$ statistical language was used to implement STA [4]. A survival tree was plotted and the survival rates at 5 and 10 years for each terminal node and their 95\% confidence interval were also calculated.

\section{Results}

A total of 1858 subjects were included in the analysis of whom 196 had a death date recorded in the database. The mean age of patients was 42 years ( $S . D=12.5$ years). Forty eight percents were male. A high proportion had contracted HIV through a heterosexual relation $(84 \%)$ and was non-French citizens (60\%). Seven percents of patients have a declared addiction (of whom 50\% with at least crack cocaine and 55\% with at least alcohol) and $17 \%$ had an HAART at enrolment. The mean CD4 count at enrolment was $343(S . D=271)$ (Table 1).

After STA calculation, the regression tree in figure 1 was obtained. A combination of 4 predictors over the 7 initial variables was used by the final tree produced by the STA: initial CD4 count, patient age, sex, and declared addictions. While overall HIV+ survival rate was $85 \%$ [CI: $83 \%-87 \%$ ] at 5 years and $81 \%$ [CI: $78 \%-85 \%$ ] at 10 years, this rate in

Table 1: Main characteristics of the study sample.

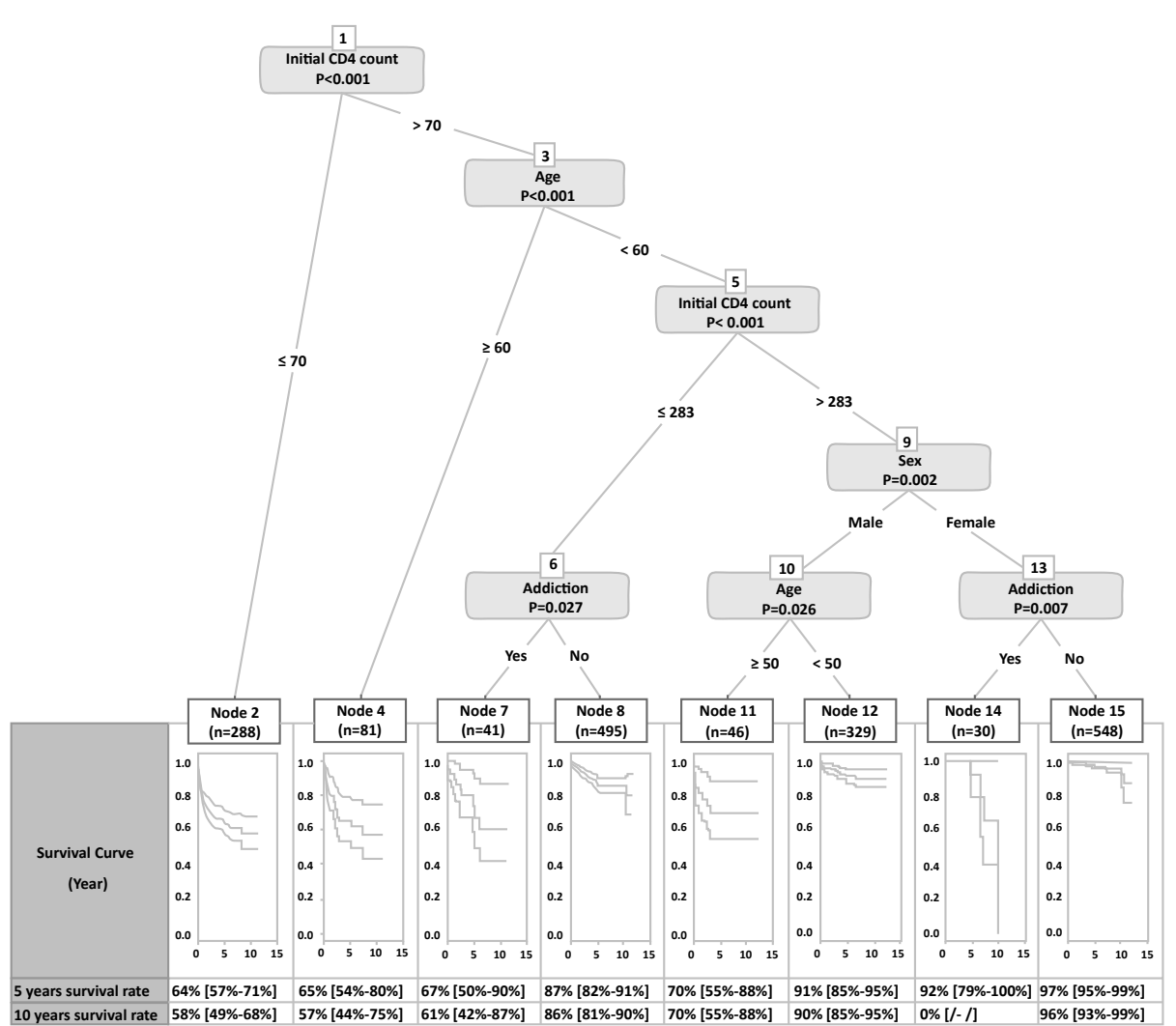

Figure 1: Survival tree for death calculated by unbiased recursive partitioning and using the predictor variables as determined by the methodology. Figures at terminal nodes show the evolution of survival rate within that branch of the tree, its $95 \%$ confidence interval, and its survival rates at 5 years and 10 years. 
HIV+ patients was divided into 8 groups according to the STA, varying from $64 \%$ to $97 \%$ at 5 years and from $0 \%$ to $96 \%$ at 10 years.

Five groups particularly at risk were identified: HIV+ patients with initial CD4 count less than 70 cells/ $\mu \mathrm{L}$ (5 years survival rate: $64 \%, 10$ years survival rate: $58 \%$ ), patient $\geq 60$ years with initial CD 4 count $\geq 70$ (5 years survival rate: 65\%, 10 years survival rate: $57 \%$ ), patients $<60$ years with initial CD 4 count $\geq 70$ and $<263$ with a declared addiction (5 years survival rate: 67\%, 10 years survival rate: $61 \%$ ), male patients aged between 50 and 60 years with an initial CD4 count $\geq 263$ (5 years survival rate: $70 \%, 10$ years survival rate: $70 \%$ ), female patients aged $<60$ years with an initial CD4 count $\geq 263$ and a declared addiction $(5$ years survival rate: $92 \%, 10$ years survival rate: $0 \%$ ).

\section{Discussion}

In survival analysis, risk stratification schemes for mortality of HIV positive patients are typically based on multivariate single failure Cox proportional hazards analysis. STA has the advantage to consider predictors in combination rather than in isolation. In this study, the clinical decision tree produced by the STA provides a simple and reliable model of the risk stratification for mortality of HIV+ patients and permits to reemphasize and identify subgroups of patients particularly at risk of an early death in French Guiana.

It is now well established that an early diagnosis of HIV infection is important to reduce the risk of HIV transmission to other partners and to reduce morbidity and mortality rates [5]. In French Guiana, it was estimated that around $40 \%$ of patients diagnosed with AIDS ignored their HIV status at the time of diagnosis [1]. Not surprisingly, a preponderant role of CD4 count at enrolment in the tree construction was retrieved and confirms that getting persons to do the HIV test and avoid late diagnosis still remains a challenge in French Guiana [1]. Further studies have thus to be conducted to better identify barriers to testing and collect evidence to improve testing in French Guiana [6].

A second important point reemphasised in this analysis is that additional efforts to reduce the high mortality of addicted patients are required in French Guiana. A large part of addictive HIV+ patients were alcohol dependent (55\%) or crack users (50\%). In French Guiana, crack cocaine and alcohol consumption are both increasing problems with serious social and health consequences [7]. Recent studies have strengthened the argument for an association between cocaine and alcohol use and acceleration of HIV disease [7-9]. The present study clearly shows the magnitude of the consequences of crack cocaine and alcohol on death of HIV+ patients in French Guiana.

In addition, the use of STA analysis revealed a new specific subgroup particularly at risk of death in French Guiana never mentioned in the literature: HIV+ female patients with a declared addiction. They represented the subgroup with the most compromised survival at 10 years $(0 \%)$. Some potential processes that could account for this gender specificity could be greater sexual risk taking and insecurity, poorer diet and nutrition, substandard living conditions, a lower use of the medical care system, and other social mechanisms [8]. Furthermore, due to the fact that most female addictions described in French Guiana concerned crack or alcohol consumption, another alternative hypothesis could be that the adverse effects of cocaine or alcohol on the immune system may vary by gender [10]. Further studies should elucidate the potential pathways of this gender specificity in French Guiana.

Our results have several limitations. First, a bias consecutive to a possible under reporting of HIV-related deaths occurring outside of hospital could not be excluded in this analysis. Furthermore, this analysis cannot control for the multiple potential confounders related to predictors (especially for addiction). Self reporting of addictions also underestimates the true prevalence of addictions. However, the present study, by using an alternative statistical method to analyze survival data, has the merit to reemphasize the magnitude of the consequences of addiction on survival of HIV+ patient, a public health problem which is at present met with scarce resources in French Guiana (few existing resources mostly located in cities, no real political involvement,...). It points that HIV+ females with a declared addiction are particularly at risk of death in French Guiana. This knowledge should lead to strengthening prevention and care in this particularly vulnerable segment of the HIV positive population.

\section{References}

1. Nacher M, El Guedj M, Vaz T, Nasser V, Randrianjohany A, et al. (2005) Risk factors for late HIV diagnosis in French Guiana. AIDS 19: 727-729.

2. Nacher M, Huber F, El Guedj M, Vaz T, Magnien C, et al. (2007) Risk factors for death among patients in French Guiana: 1996-2005. HIV Med 8: 472-474.

3. De'ath G, Fabricius KE (2000) Classification and regression trees: a powerful yet simple technique for ecological data analysis. Ecology 81: 3178-3192.

4. Hothorn T, Hornik K, Zeileis A (2006) Unbiased Recursive Partitioning: A Conditional Inference Framework (2006). Journal of Computational and Graphical Statistics: 651-674

5. Girardi E, Sabin CA, Monforte AD (2007) Late Diagnosis of HIV Infection: Epidemiological Features, Consequences and Strategies to Encourage Earlier Testing. J Acquir Immune Defic Syndr 46: S3-8.

6. Hanf M, Bousser V, Parriault MC, Van-Melle A, Nouvellet ML, et al. (2011) Knowledge of free voluntary HIV testing centres and willingness to do a test among migrants in Cayenne, French Guiana. AIDS Care 23: 476-485.

7. Cook JA, Burke-Miller JK, Cohen MH, Cook RL, Vlahov D, et al. (2008) Crack cocaine, disease progression, and mortality in a multicenter cohort of HIV-1 positive women. AIDS 22: 1355-1363.

8. Lucas GM, Gebo KA, Chaisson RE, Moore RD (2002) Longitudinal assessment of the effects of drug and alcohol abuse on HIV-1 treatment outcomes in an urban clinic. AIDS 16: 767-774

9. Nacher M, Adenis A, Hanf M, Adriouch L, Vantilcke V, et al. (2009) Crack cocaine use increases the incidence of AIDS-defining events in French Guiana. AIDS 23: 2223-2226.

10. Cabral GA (2006) Drugs of abuse, immune modulation, and AIDS. J Neuroimmune Pharmacol 1: 280-295. 